Rabaska

Revue d'ethnologie de l'Amérique française

\title{
Mémoires et thèses
}

\section{Carmen d'Entremont et Gisèle Thériault}

Volume 11, 2013

URI : https://id.erudit.org/iderudit/1018553ar

DOI : https://doi.org/10.7202/1018553ar

Aller au sommaire du numéro

Éditeur(s)

Société québécoise d'ethnologie

ISSN

1703-7433 (imprimé)

1916-7350 (numérique)

Découvrir la revue

Citer ce document

d’Entremont, C. \& Thériault, G. (2013). Mémoires et thèses. Rabaska, 11, 259-273. https://doi.org/10.7202/1018553ar d'utilisation que vous pouvez consulter en ligne.

https://apropos.erudit.org/fr/usagers/politique-dutilisation/ 


\section{Annuel}

\section{Mémoires et thèses}

\section{Carmen D'Entremont et Gisèle ThÉRIAUlt}

Université Sainte-Anne

NDLR - On trouvera dans cette rubrique les résumés de quinze mémoires et de huit thèses reliés au domaine de l'ethnologie de l'Amérique française. Ces travaux ont été préparés par des étudiants inscrits à une dizaine de programmes - ethnologie et patrimoine, anthropologie, études littéraires, linguistique, sémiologie, communication, sociologie, géographie et environnement - de huit universités canadiennes : on dénombre sept études à l'Université Laval (6 M.A et 1 Ph. D.), six à l'Université de Montréal (5 M.A et $1 \mathrm{Ph}$. D.), trois à l'Université de Moncton (1 M.A et $2 \mathrm{Ph}$. D.), deux à l'Université du Québec à Montréal (1 M.A et $1 \mathrm{Ph}$. D.) et à l'Université d'Ottawa (2 Ph. D.), et une à l'Université du Manitoba (1 M.A), à l'Université York (1 Ph. D.) et à l'Université de Sherbrooke (1 M.A).

Apetrei, Amelia-Elena. Analyse sémio-narrative d'un corpus de contes merveilleux du Québec. Mémoire (M. A.), Université de Montréal, 2011. 1-117 p. [Direction : Guy Laflèche].

Ce mémoire propose une analyse sémio-narrative d'un corpus de dix-neuf contes merveilleux recueillis auprès des conteurs canadiensfrançais et canadiens-hurons par l'anthropologue Charles-Marius Barbeau, au début du $x^{e}$ siècle. Après avoir passé en revue les principales théories dans l'approche sémiotique du conte à partir de Vladimir Propp jusqu'à nos jours, nous avons procédé à une étude narrative du corpus selon la méthode du grand folkloriste russe : cela nous a permis d'en montrer la valeur exceptionnelle mais aussi les limites. Nous avons constaté ainsi que le travail sur un corpus inédit peut mener à l'identification de nouveaux éléments dans la structure du conte merveilleux. En poursuivant nos recherches sur les dix-neuf contes recueillis par Barbeau, nous passons du schéma linéaire, syntagmatique de Propp, aux modèles logiques de ses continuateurs, particulièrement celui élaboré par Guy Laflèche. Nos analyses ont mis en évidence non seulement le fait que la structure en miroir est un modèle valide auquel une partie des contes 
merveilleux se plie parfaitement (d'emblée l'exclusivité du schéma de Propp est mise en question, sinon infirmée), mais aussi que tout conte merveilleux est exceptionnellement organisé, structuré (ce qui confirme la théorie de Claude Bremond selon laquelle le récit narratif est une alternance de dégradations et d'améliorations). Enfin, la dernière partie du mémoire est une analyse sémiodiscursive de notre corpus qui, au lieu d'être une simple accumulation de listes de mots, d'expressions et de figures, tente d'aborder la structure idéologique, grâce à une étude en parallèle d'un conte du corpus et de deux versions littéraires.

Asselin, Geneviève. Illustration d'une pratique communicationnelle du subjectif: présentation et récit de soi dans les sites de partages de photographies. Mémoire (M. A.), Université du Québec à Montréal, 2008, vii-111 p. [Direction : Luce Des Aulniers].

Ce mémoire rend compte d'une recherche de type exploratoire sur les formes d'exposition de soi dans les sites de partage de photos tels que Flickr.com et Fokti.com. Plus précisément, nous y explicitons les pratiques de présentation et mise en récit de soi dans ces sites que nous pouvons désigner comme sites générés par les usagers. Notre démarche s'avère relativement novatrice puisqu'elle témoigne de l'émergence d'un nouvel objet communicationnel et qu'elle est le fruit d'une transversalité des disciplines. Notre objet d'étude nous permet d'aborder les codifications rendues possibles par certains aspects techniques de ces sites et d'explorer les significations communicationnelles et culturelles des sites de partage de photos. Ce faisant, nous examinerons les identités narratives véhiculées par les interventions et manipulations des usagers sur les sites choisis par une grille d'analyse socio-historique tout en appliquant des procédés d'observation empruntés à l'ethnologie. Ceci dans le but d'une compréhension à visée globale des principaux traits d'une culture observable à travers sa manifestation virtuelle. Les études de cas présentées ici nous permettent à la fois de comprendre l'usage d'un nouveau média et de mieux saisir comment son utilisation fabrique une identité individuelle, mais aussi un modèle d'interaction formant une société virtuelle. Elles permettent également de comparer cette pratique d'autodiffusion au journal intime, un genre littéraire dont l'évolution est particulièrement observable dans les différentes phases de la modernité. Ainsi, la réflexion s'attarde autour de l'identité et de la culture de l'individualité dans la phase actuelle de la modernité, et présente les sites de partage de photos comme objets porteurs et éloquents d'une culture contemporaine. 
Beaulieu, Julie-Éliane. L'Ethnodesign. Un dialogue entre l'artisanat et le design contemporain. Mémoire (M. A.), Université Laval, 2012, viii-121 p. [Direction : Jocelyne Mathieu].

L'exploration de la notion d'ethnodesign permet de découvrir de multiples facettes qui révèlent une polysémie du terme, voire une ambiguïé du concept. Celui-ci s'inscrit dans un mouvement entre le passé et le présent, l'artisanat et le design, le fait main, la préoccupation pour le développement durable et même l'apport de l'industrie. Ce va-et-vient s'exprime dans divers objets dont les caractéristiques se superposent, se croisent, et parfois se confondent. Le design s'inspire de différentes manières de l'artisanat et de ses savoir-faire ; en revanche l'artisanat utilise le design afin d'actualiser les créations. Les sources consultées, magazines, observation de projets et expériences de voyages confirment et vérifient largement l'hypothèse selon laquelle l'ethnodesign est hétérogène et que ses composantes s'articulent de différentes manières selon les contextes et les acteurs.

Brazeau, Nicolas. Ethnographie de l'usage rituel d'ayahuasca au Québec. Mémoire (M. A.), Université de Montréal, 2012, vi-112 p. [Direction : Robert Crépeau].

L'ayahuasca est une décoction de plantes originaires de la forêt amazonienne. Elle contient la vigne nommée ayahuasca (Banistereopsis caapi) et un arbuste (Psychotria viridis). Ces plantes contiennent des substances psychoactives. Respectivement il s'agit de l'harmine et de la diméthyletryptamine (DMT). Ce mélange est utilisé par plusieurs peuples autochtones du bassin amazonien depuis une époque qui précède la Conquête. L'ayahuasca est utilisée par ces peuples à des fins chamaniques. Au début $\mathrm{du} \mathrm{Xx}^{\mathrm{e}}$ siècle, la demande en caoutchouc a engendré une migration de travailleurs dans ces régions. Un de ces travailleurs d'origine africaine est entré en contact avec cette substance. De croyance chrétienne, il a interprété son expérience comme une rencontre avec le divin. Ceci l'a amené à fonder dans les années 1930 une église syncrétique nommée Santo Daime. Depuis, l'utilisation rituelle d'ayahuasca est répandue dans le monde. Aujourd'hui, on retrouve au Québec des groupes faisant un usage rituel d'ayahuasca. Ce mémoire est une ethnographie d'un groupe actif au Québec, qui a fait l'objet d'observations participantes durant l'été 2010. L'étude a démontré que la participation aux cérémonies d'ayahuasca engendre des prises de conscience et que le travail de groupe en permet l'intégration dans le quotidien des participants. De plus, la structure rituelle garantit un usage non abusif. 
Comeau, Philip. A Window on the Past. A Move toward the Future: Sociolinguistic and Formal Perspectives on Variation in Acadian French. Thèse (Ph. D.), Université York, 2011, xviii-282 p. [Direction : Ruth King].

Cette thèse examine la variation dans le choix du mode et dans l'expression de la référence temporelle au futur dans une variété conservatrice du français acadien. Les données proviennent de deux corpus recueillis à la baie Sainte-Marie (Nouvelle-Écosse) dans les villages de Grosses-Coques et de Météghan. J'intègre deux approches, notamment la sociolinguistique variationniste et le minimalisme. L'analyse pour le choix du mode (la variation entre le subjonctif et les autres modes) montre qu'il y a peu de variation et que le subjonctif est lié à la présence d'un trait sémantique, l'assertion. L'analyse du futur (la variation entre le futur fléchi et le futur périphrastique) montre que le futur fléchi est utilisé plus souvent qu'en variétés laurentiennes. De plus, le choix des variantes du futur est conditionné par la référence temporelle : les événements devant se produire à proximité du moment de l'énoncé favorisent le futur périphrastique. L'analyse formelle suggère que la variation linguistique peut se produire à deux endroits : avant et après la composante syntaxique. L'étude de ces deux variables démontre que le système linguistique du français acadien s'avère plutôt conservateur, surtout en comparaison avec d'autres variétés du français contemporain.

Curé, Mélanie. Pas juste une question de langue : l'identité nationale et l'exiguïté littéraire dans les récits franco-manitobains et acadiens. Mémoire (M. A.), Université du Manitoba, 2012, i-82 p. [Direction : Étienne Beaulieu].

Les institutions littéraires francophones du Canada cherchent à répondre à la même question que se posent les communautés dont elles font partie. Qui sommes-nous ? Cette étude fait ressortir les tendances et les caractéristiques des littératures francophones minoritaires en s'appuyant sur l'œuvre de deux auteurs, J.R. Léveillé au Manitoba francophone et Antonine Maillet en Acadie. Cette analyse éclaire les approches différentes que prennent les écrivains face à l'écriture. Ceux-ci se rangent soit du côté de la tradition, de l'histoire nationale et de la mémoire, soit du côté du moderne, du nouveau et du rejet du passé. En s'appuyant sur les théories d'Anne-Marie Thiesse, de François Paré et d'Éric Méchoulan, cette étude remarque que les tendances littéraires se manifestent à travers l'écriture et la réécriture de l'histoire, l'intertextualité, la langue et l'inclusion (ou non) des référents culturels et mémoriels. 
HéBert, Christine. Là où la main de l'homme n'a jamais mis le pied. Une netnographie des Duggies. Mémoire (M. A.), Université Laval, 2012, viii-126 p. [Direction : Martine Roberge].

Issu des universités britanniques, le courant des cultural studies s'est intéressé à l'étude de la culture populaire, dont celle des fans. Au Québec, il existe peu d'études reliées à ce domaine, et aucune à notre connaissance sur la série de science-fiction humoristique Dans une galaxie près de chez vous. Diffusée à Canal Famille (puis à VRAK-TV) de 1998 à 2001, la série a connu une grande popularité qui s'est confirmée par la sortie de deux films faisant suite à l'aventure télévisée. Ce mémoire se concentre sur l'étude d'une communauté de fans de cette série, réunis dans un forum nommé Dans une galaxie Online (ou DugOnline) fondé en 2004 et toujours actif à ce jour. Nous nous intéressons en particulier à la socialisation exercée entre les membres du forum (aussi nommés Duggies) ainsi qu'aux raisons expliquant la présence de membres toujours actifs huit ans après leur inscription. Cette étude fait appel à la netnographie, soit l'ethnographie de l'Internet. Nous avons opté pour un croisement des sources, qu'elles soient numériques, orales ou manuscrites. Par l'apport des participants aux entrevues orales, l'analyse révèle entre autres les étapes franchies par un membre, depuis son inscription sur le forum au maintien des liens avec les autres membres. En plus d'être un lieu de socialisation, le forum permet le partage d'allusions reliées à la série, il est le lieu d'expression d'un culte voué aux acteurs de la série ainsi que le point de départ de rituels et de fêtes célébrés par les membres de la communauté. En conclusion, nous expliquons comment le forum, formé d'individus de différents milieux socioéconomiques, se transforme en communauté. Cette dernière, unie par l'intérêt pour une même série télévisée, aura finalement transformé la vie de ses membres.

Joubert, JoAnie. Discours et identité d'un migrant canadien-français au $X I X^{e}$ siècle. La trajectoire de Sam Gravel. Thèse (Ph. D.), Université d'Ottawa, 2012, 1-161 p. [Direction : France Martineau].

Dans cette étude, nous examinons la trajectoire d'un migrant canadienfrançais au XIX ${ }^{\mathrm{e}}$ siècle, Sam Gravel, ainsi que les changements identitaires qu'il subit en fonction des lieux de migration qu'il atteint. Sam se rend en Nouvelle-Angleterre en 1882 pendant quelques mois. Il revient brièvement au Québec puis immigre dans l'Ouest canadien en 1883 et y demeurera jusqu'en 1891. Il s'établira ensuite dans le Midwest américain jusqu'en 1897. Il rentrera finalement au Québec et s'installera dans le village de Somerset en 1897. Il décède en 1899 d'une noyade à Québec. 
C'est en analysant son parcours migratoire, son mode d'établissement et son discours que nous pouvons déterminer comment s'insère sa migration dans les courants de migration du XIX ${ }^{\mathrm{e}}$ siècle. Est-ce que ses choix sont représentatifs de la majorité des migrants de l'époque ? Comment ces choix influencent-ils sa perception des autres groupes ethniques ainsi que ses valeurs religieuses et familiales? Sam tient un discours qui se rapproche beaucoup de celui des Canadiens français de l'époque alors qu'il se trouve dans l'Ouest canadien et tend à s'en dégager alors qu'il atteint le Midwest américain.

JoURDAIN, VéroniQue. Quête spirituelle et réconciliation identitaire : une ethnographie de la Communauté des druides du Québec [CDQ]. Mémoire (M. A.), Université de Montréal, 2013, iii-155 p. [Direction : Deirdre Meintel]. Au cours du $\mathrm{Xx}^{\mathrm{e}}$ siècle, le Québec a connu un bouleversement identitaire et religieux lorsqu'une majorité de Québécois a délaissé les prescriptions doctrinales catholiques, la religion identitaire du Québec, qui encadrait leur vie et s'imposait en tant que détentrice du « code exclusif du sens ». Pour combler la perte de repères engendrée par les bouleversements décrits précédemment, plusieurs individus se tournèrent vers d'autres formes de spiritualité, soit en se convertissant à un nouveau système religieux ou en s'engageant dans une quête spirituelle. Certains Québécois, au cours de leur quête, ont alors découvert le druidisme, une spiritualité fondée sur le désir de retour à l'ancienne religion païenne des Celtes, et l'ont intégré en tant que composante principale ou secondaire de leur spiritualité. Le but de ce mémoire est d'apporter quelques éléments d'explication à la présence du druidisme au Québec. Nous soulignons l'importance du contexte moderne dans la quête de sens amorcée par l'adepte de paganisme celte, car ce contexte semble avoir servi de catalyseur dans le processus de découverte du druidisme pour ces Québécois. Puis, nous mettons l'accent sur les raisons identitaires mises de l'avant par la CDQ pour justifier leur choix d'intégrer le druidisme à leur cheminement spirituel. Pour le druidisant, cette religion vient à la fois répondre à des besoins spirituels et combler un vide identitaire.

Julien, Anne. Les Festivals francophones en Ontario : vecteurs de la vitalité culturelle d'une communauté minoritaire - une étude de cas multiples. Thèse (Ph. D.), Université de Montréal, 2012, vi-338 p. [Direction : Marcel Fournier]. Cette recherche consiste en un examen du rôle du festival dans une communauté linguistique en contexte minoritaire confrontée à des enjeux spécifiques tels que l'assimilation, l'exogamie, la diglossie, l'inégal accès 
aux services dans sa langue, de même que l'accès limité aux produits culturels de son groupe linguistique. Le cas des festivals francophones en milieu minoritaire sert à interroger concrètement et empiriquement les possibilités, les conditions, les limites et les contraintes de cette forme particulière d'événement en tant que vecteur de la vitalité culturelle d'une communauté minoritaire. Le festival est aussi exploré en lien aux grandes finalités de développement culturel et de démocratie culturelle, soit des mécanismes culturels à la source des politiques culturelles modernes, ainsi qu'en lien à son rôle aux niveaux de l'affirmation et de la consolidation identitaires. Une étude de cas multiples a été menée afin de comparer et d'analyser trois festivals francophones en milieu minoritaire ontarien qui sont mis sur pied dans différentes régions, et ont des objectifs à la fois similaires et différenciés : La Nuit sur l'étang (Sudbury) ; le Festival franco-ontarien (Ottawa); et le Festival du Loup (Lafontaine-Pénétanguishene). Cette recherche présente, par le biais de sources documentaires, d'observations sur le terrain et d'entretiens auprès des organisateurs, des festivaliers et des artistes de ces événements, les perceptions qu'en ont et l'utilisation qu'en font ces différentes catégories d'acteurs. Elle fait état de la contribution du festival au niveau des mécanismes culturels et permet de déterminer si les acteurs le perçoivent comme un enjeu important pour la francité. Bref, l'ensemble des contributions qu'apportent les festivals francophones en Ontario montrent en quoi ces événements agissent comme des vecteurs de la vitalité culturelle d'une communauté minoritaire.

Lacroix, Marie-Élaine. La Mise en valeur du littoral du secteur est de la réserve du Parc national du Canada de l'Archipel-de-Mingan en s'appuyant sur le patrimoine culturel et autochtone de la Première Nation innue de Nutashkuan. Mémoire (M. A.), Université de Sherbrooke, 2013, iii-100 p. [Direction : Denis Dufour].

Parcs Canada effectue la gestion de ses parcs nationaux en protégeant et en mettant en valeur les patrimoines naturels et culturels qui en font partie afin qu'ils demeurent intacts pour les générations actuelles et futures. Pour que cette gestion s'effectue en harmonie avec les communautés locales, notamment avec les Premières Nations, une collaboration maximale doit être entretenue. La réserve du Parc national de l'Archipel de Mingan est une aire protégée où la Première Nation innue de Nutashkuan souhaite participer pleinement à la gestion et à la planification du territoire. Dans ce contexte, l'objectif principal de cet essai consiste à poser un diagnostic sur la présence et les connaissances actuelles et passées de cette nation 
afin de les intégrer à la gestion et à la mise en valeur du secteur est de la réserve. L'atteinte de cet objectif se base sur une analyse de différents aspects culturels de la communauté de Nutashkuan afin de déterminer les éléments les plus aptes à être mis en valeur et les moyens pour y parvenir. Les résultats de l'analyse permettent d'élaborer des recommandations sur les activités à privilégier dans la réserve, les éléments de la culture innue à mettre en valeur et les façons de les promouvoir sur le marché.

LAmBert-Pellerin, CASSANDRE. L'Éducation continue des femmes en réseau : l'exemple de l'Association féminine d'éducation et d'action sociale (AFÉAS). Mémoire (M. A.), Université Laval, 2013, ii-116 p. [Direction : Jocelyne Mathieu].

Lieux d'expression féminine à l'extérieur du cadre familial, plusieurs associations ont contribué, chacune à sa façon, à l'émancipation des femmes. Si certaines sont demeurées sur des positions plutôt conservatrices, d'autres ont revendiqué des changements sociaux. L'Association féminine d'éducation et d'action sociale (AfÉAs) se situe à la jonction de ces deux courants de pensées. Sans vouloir renier le rôle traditionnel de la femme, l'AfÉAs se prononce sur la place publique et milite depuis quarante-cinq ans pour faire bouger les choses. Le dossier des femmes collaboratrices et celui de la reconnaissance des travailleuses au foyer sont le cheval de bataille de l'Association. L'AféAs place au cœur de ses actions la parole des femmes, traditionnellement informelle, liée à la transmission orale des savoirs et savoir-faire. Au sein de l'AfÉAs, la notion d'éducation populaire des femmes adultes est centrale parce qu'elle fait partie de sa mission principale. Ce mémoire cherche à comprendre comment l'expérience des femmes au sein de l'AfÉAs favorise la formation de réseaux de communication, d'affinités, de mobilisation et de soutien, qui transmettent de l'information utile au mieux-être de ses membres, et dont les effets bénéfiques s'étendent au-delà des AfÉAs locales. Cette recherche s'appuie sur les témoignages oraux de onze membres de l'AfÉAs du Centre-du-Québec rencontrées en mai et juin 2011.

LANDRY, Jonathan. Étude de représentations linguistiques de jeunes Acadiennes et Acadiens en milieu scolaire : vers un éveil à sa propre langue? Mémoire (M. A.), Université de Moncton, 2012, ix-235 p. ill. [Direction : Annette Boudreau et Laurence Arrighi].

Cette recherche, de type ethnosociolinguistique, examine la façon dont un cours consacré à la variation linguistique permet le développement d'une réflexion sociolinguistique et métalinguistique. Ce cours, « Parler 
acadien », dispensé au district scolaire 1 du Nouveau-Brunswick, met de l'avant une variété régionale (ses formes, ses causes et ses conséquences, tant externes qu'internes) et entend faire prendre conscience aux jeunes participants des représentations qu'ils se font de leur langue et de celle des autres. L'étude s'inspire des programmes européens d'éveil aux langues, une approche pédagogique interculturelle qui a pour principale mission de sensibiliser positivement les élèves à la diversité des langues. En observant (observation participante/enquête de terrain) et en questionnant (entretiens semi-directifs) cet enseignement, je me propose d'examiner la manière dont les représentations linguistiques se manifestent chez les élèves et déterminer si un cours portant sur le parler acadien agit sur ces représentations.

LeBlanc, MéLANIE. Idéologies, représentations linguistiques et construction identitaire à la baie Sainte-Marie, Nouvelle-Écosse. Thèse (Ph. D.), Université de Moncton, 2012, vii-346 p. ill. cartes. [Direction : Annette Boudreau].

Cette thèse s'attache à décrire et à comprendre certains des processus sociaux qui entrent en jeu dans la (re)construction identitaire d'une communauté francophone en milieu minoritaire, soit une communauté acadienne du sud-ouest de la Nouvelle-Écosse connue sous le nom de la baie Sainte-Marie, dans une période de transformations sociales et économiques liées à la nouvelle économie mondialisée. Plus précisément, à travers trois espaces discursifs d'intérêts (l'école, la radio communautaire et le tourisme culturel), elle cherche à voir quel est le rôle de la langue dans ces processus en se penchant sur les luttes discursives qui rendent compte de rapports de pouvoir entre des groupes d'individus qui revendiquent le « droit » de délimiter les frontières identitaires de la communauté, selon des intérêts (d'ordre social, économique, politique) et des positionnements idéologiques particuliers. En mobilisant les concepts d'idéologies, de représentations linguistiques et de ressources langagières, la thèse cherche à montrer comment les changements sociaux et économiques s'articulent aux enjeux linguistiques et identitaires dans la communauté et comment la langue devient un terrain de revendication pour l'accès à des ressources symboliques et matérielles. Le travail s'inscrit dans une sociolinguistique critique qui permet d'aborder les pratiques linguistiques en lien avec des processus sociaux plus larges, se révélant à la fois dans le temps et dans l'espace. La démarche ethnographique a été privilégiée, de même qu'une démarche empirico-inductive, qui accorde la priorité au terrain. 
Lefebvre, Marie. Le Rôle géographique de la fête : le Congrès mondial acadien comme catalyseur identitaire et inhibiteur de frontières. Thèse (Ph. D.), Université d'Ottawa, 2012, i-226 p. [Direction : Anne Gilbert].

Dans la foulée des débats entourant la définition et la compréhension de l'Acadie, dans ses dimensions identitaires et territoriales, cette thèse se veut un regard analytique sur l'adéquation entre la fête, le territoire et l'identité. Par l'étude du $4^{\mathrm{e}}$ Congrès mondial acadien tenu en août 2009 dans la péninsule acadienne au Nouveau-Brunswick, nous cherchons à mieux cerner le rôle géographique de la fête, de même que son importance dans le développement des collectivités minoritaires comme l'Acadie. Ainsi, en étudiant trois terrains différents, soit le terrain géographique tel que dévoilé dans le jeu politique d'échelles performé par les organisateurs, celui des identités telles qu'elles sont exprimées dans le discours des participants et celui de l'action et des performances spatiales des gens et des paysages, nous tentons de démontrer que le CMA est une fête qui témoigne certes de l'ambivalence de l'Acadie et de nos sociétés contemporaines, mais dans un contexte qui se veut essentiellement culturel, unificateur et rassembleur. En ce sens, il se constitue un événement qui permet à la communauté de se vitaliser, de se renouveler et de s'ancrer non plus uniquement dans un passé trouble, mais dans un présent et un avenir riches de possibilités. Sans nécessairement trancher le débat, cette analyse se veut porteuse d'une réflexion positive pour la communauté acadienne, tout en permettant d'élargir le propos théorique et méthodologique sur la fête en géographie.

Matte, Isabelle. Rites de passage en contexte moderne : l'exemple de la scène musicale underground montréalaise. Mémoire (M. A.), Université Laval, 2000, 130 p. [Direction : Bernard Arcand et Jean-Jacques Chalifoux].

Ce mémoire porte un regard sur la société « occidentale » moderne, particulièrement sur le passage à l'âge adulte et sur la période que nous nommons adolescence. Nous avons pour ce faire enquêté sur un regroupement de jeunes basé sur une culture musicale, la scène underground montréalaise. Le point central de cette recherche est l'étude du rituel, particulièrement les rites de passage et les rites d'inversion : nous tentons de voir comment se construisent certaines façons de passer à l'âge adulte à l'intérieur de sociétés industrialisées et fragmentaires, en prenant Montréal et le Québec en exemple. Notre enquête de terrain a été menée de juillet à novembre 1998 auprès des musiciens et des fans de cette scène musicale. L'exposé qui en résulte se veut le résultat d'une recherche exploratoire plutôt que la démonstration d'un argument précis, 
surtout à cause des travaux peu nombreux sur les rites de passage de la jeunesse actuelle.

Matte, Isabelle. Sortir de la religion. Spécificités d'une sécularisation catholique au Québec et en Irlande. Expériences du "Celtic Tiger» et de la Révolution tranquille. Doctorat (Ph. D.), Université Laval, 2013, i-421 p. [Direction : Sylvie Poirier et Nancy Schmitz].

Cette thèse porte sur une comparaison entre le déclin rapide des pratiques catholiques dans l'Irlande du Celtic Tiger (1994-2008) et dans le Québec de la Révolution tranquille (1963-1980). Elle tend à démontrer que ces deux contextes de réelle effervescence politique, économique, sociale et culturelle, ont constitué un passage fondamental dans l'imaginaire collectif de ces deux sociétés, et que la perte d'autorité de l'Église catholique lors de ces périodes est constitutive de ce passage. De cette « sortie de la religion » ont résulté des sociétés profondément transformées au cœur même du sens commun. Dans les deux cas, c'est la jeune génération qui, favorisée par la démographie, a le plus contribué à faire chuter la pratique religieuse. L'abandon de la messe dominicale, point d'ancrage d'une certaine religiosité traditionnelle basée sur l'appartenance à la famille et à la paroisse, est le marqueur de ce déclin. L'étude compare les contextes historiques du Québec et de l'Irlande afin d'y montrer l'influence de la conquête britannique sur le type de catholicisme qui s'y est élaboré. Face à une difficile conquête pour la souveraineté politique, l'Église est devenue l'institution identitaire par excellence. Avec l'ultramontanisme du XIX ${ }^{\mathrm{e}}$ siècle, l'Irlande, tout comme le Québec, connaît un renouveau religieux initié par une solide prise en charge des domaines de la santé et de l'éducation par le clergé et les ordres religieux. Cette situation incite à une nouvelle ferveur qui sera au cœur d'un type de religiosité qui englobe toutes les sphères de l'existence. Plus tard, au moment où les normes de vie basculent et où les changements s'accélèrent pendant les périodes étudiées, l'Église catholique devient soudainement désuète pour la majorité des gens. Il s'agit alors de s'en extirper. Inspirée par Max Weber et basée sur des observations et des données de terrains effectués au Québec et en Irlande sur une période de 20 ans, cette thèse explore et trace les contours de ce qui semble être une sécularisation typiquement catholique.

Philippe-Shillington, Julie. Les Valeurs traditionnelles canadiennesfrançaises dans les pratiques familiales des Franco-Ontariens. Mémoire (M.A.), Université Laval, 2013, xi-218 p. [Direction : Jocelyne Mathieu]. 
Pour étudier les valeurs traditionnelles canadiennes-françaises dans les pratiques familiales des Franco-Ontariens du nord-est de l'Ontario dans un ménage mixte francophone-anglophone, il faut essentiellement pénétrer au cœur de sa vie quotidienne. Une telle étude ne peut être possible sans considérer le contexte historique qui permet non seulement de connaître les sujets d'étude, mais aussi d'authentifier son passé. Ce travail gravite autour de la décennie des années 1960, période reconnue pour ses réformes entraînées par la Révolution tranquille au Québec et, par effet d'entraînement, chez certains de ses voisins. L'objet de l'étude veut appréhender le phénomène de la conciliation des valeurs traditionnelles dans les pratiques coutumières plus particulièrement depuis les bouleversements de cette période. Une enquête orale de type ethnographique sur le terrain auprès de deux familles intergénérationnelles de trois couples permet une importante collecte de données. Les récits de vie des informateurs des trois générations dévoilent de façon exemplaire le visage traditionnel de l'Ontario français du nord de la province en faisant transparaître sa vitalité quotidienne.

PréBolin, Estelle. Les Cours de danse africaine à Montréal, émergence d'une production socioculturelle et esthétique. Mémoire (M. A.), Université de Montréal, 2011, 5-133 p. [Direction : Bob White].

Ce mémoire traite de l'enseignement de la danse africaine en France et au Canada. Cette recherche a débuté en 2007, lorsque l'auteur a participé à un échange étudiant. À la fin de cette expérience, l'auteur en était arrivé à la conclusion que la danse africaine au Québec était abordée comme un bien de consommation ou une production socioculturelle relevant de l'imaginaire. La présente analyse explore les avantages et les limites de l'approche méthodologique adoptée par l'anthropologue (qui est, dans ce cas-ci, une ancienne danseuse classique) et les conditions de la rencontre entre les Africains et les Occidentaux par la danse. Tandis qu'il reste à démontrer que les critiques postmodernes de l'art de masse s'appliquent dans ce cas-ci, l'analyse montre clairement que l'on trouve dans les cours de danse africaine au Québec une forme de conscience professionnelle. Les critiques de la danse et d'autres formes de démocratisation artistique tendent à se confondre dans la peur du discours populaire. L'objectif principal de cette recherche est par conséquent d'établir les limites du fétichisme par rapport à la danse africaine et d'explorer en détail les implications de la « hantise du Troisième Homme » communiquée par la recherche ethnographique et l'analyse anthropologique. 
Provencher St-Pierre, Laurence. La Collecte de l'objet contemporain. L'exemple du Musée de la civilisation de Québec. Mémoire (M. A.), Université Laval, 2012, iv-121 p. ill. [Direction : Jocelyne Mathieu].

Ce mémoire porte sur la collecte des objets contemporains en contexte muséal, par l'exemple du Musée de la civilisation, et pose un regard sur les pratiques entourant la collecte du contemporain dans cette institution, de son ouverture en 1988 à aujourd'hui ; elle fait ressortir les différentes étapes qui permettent à l'objet récent d'acquérir le statut d'objet de musée. Après sa sélection, sa documentation, son acquisition ainsi que sa prise en charge permettant sa conservation à long terme, l'objet contemporain intègre la collection nationale. Bien que son parcours soit similaire à celui traversé par les objets plus anciens, il s'en distingue par les enjeux et les limites inhérentes à sa collecte, l'objectif du Musée étant d'encourager son acquisition afin de constituer des collections qui représenteront demain la société d'aujourd'hui. En faisant ressortir le processus d'intégration des objets à la collection, cette étude met également en lumière le rôle des conservateurs dans le développement des collections muséales.

Richard, MoniQue. Comprendre le phénomène du rôle de passeur culturel en milieu francophone minoritaire du Nouveau-Brunswick par le témoignage de membres du personnel enseignant. Thèse (Ph. D.), Université de Moncton, 2012, ii-268 p. [Direction : Jeanne d'Arc Gaudet et Réal Allard].

Cette thèse aborde l'expérience du rôle de passeurs culturels, vécue par des enseignantes et un enseignant du primaire dans des écoles de langue française en milieu urbain anglodominant du Nouveau-Brunswick, pour comprendre leur réalité relativement à la double mission de réussite scolaire et de développement de la langue et de la culture françaises des élèves en milieu francophone minoritaire. Le fruit du travail d'analyse phénoménologique-herméneutique a permis de circonscrire les éléments les plus révélateurs afin de saisir le sens général du phénomène relié à ce rôle. Parmi ceux-ci, le cheminement identitaire, culturel et professionnel des enseignantes et de l'enseignant montre qu'une diversité d'expériences socialisantes, dans des contextes socioculturels variés, a façonné leur identité et leur rapport à la langue et à la culture françaises, et les a sensibilisés et motivés, à différents degrés, à s'engager envers la communauté francophone et dans leur rôle de passeurs culturels. Par ailleurs, l'analyse-interprétation dévoile que les participantes et le participant ont des principes et convictions qui les guident par rapport à leur rôle et à la mission de l'école française. Ces individus cherchent alors à créer un espace francophone à l'école où les jeunes ont l'occasion de s'exprimer 
en français et de développer leurs compétences langagières. Alors que certains jouent leur rôle de passeurs culturels avec aisance et plaisir et éprouvent une grande satisfaction à intégrer des pratiques culturelles régulières, diversifiées et réflexives afin de contribuer à la construction identitaire francophone, d'autres vivent de l'incertitude et même de l'insécurité relativement à leur rôle et n'arrivent pas à intégrer une dimension culturelle de façon planifiée. Cette thèse offre une compréhension renouvelée du phénomène du rôle de passeur culturel en milieu francophone minoritaire, ainsi que certaines pistes de réflexion découlant des témoignages des enseignantes et de l'enseignant qui pourraient contribuer à orienter le soutien et l'accompagnement leur permettant de jouer pleinement leur rôle de passeurs culturels.

SÉGuin, Madeleine. Entre danser pour soi et danser pour les autres : la construction de l'expérience dans le processus de socialisation des danseurs de tango argentin de Montréal. Mémoire (M. A.), Université de Montréal, 2012. iv-155 p. [Direction : Jorge Pantaleon].

Cette étude ethnographique porte sur le réseau social du tango argentin à Montréal, les valeurs esthétiques du tango, l'interprétation de l'expérience de la danse acquise au cours du processus de socialisation et les différents agents de socialisation. Dans un contexte de réappropriation et de déterritorialisation d'un savoir-faire comme le tango, les références symboliques relatives à son contexte d'origine, son imaginaire collectif et son contexte sociohistorique sont moins accessibles. Ceci semble appeler chez les danseurs montréalais une sorte de surdétermination de l'expérience immédiate, concrète et interactive de la danse dans la construction d'un sens collectif. Cette recherche s'intéresse plutôt à la transposition dans le réseau montréalais de certaines normes esthétiques en lien avec les modalités interactives de la danse. Ces normes esthétiques induisent une attention particulière portée à la qualité de l'expérience se reflétant dans les pratiques et les discours. Ces valeurs esthétiques remontent au contexte d'origine du tango et à la rencontre entre différents univers socialisateurs transnationaux à différentes étapes de son évolution. De ce type de rencontres « transesthétiques », s'est développé un dualisme entre le danser pour les autres et le danser pour soi, dans les représentations et la mise en forme d'une expérience dansée. À Montréal, on observe la cohabitation de ces deux systèmes de valeurs en apparence antagonistes où, d'une part, est valorisée l'exhibition du soi et, d'autre part, sont priorisées l'intériorité et l'expérience subjective. En bref, ce mémoire explore les relations complexes qui existent entre les processus culturels 
et leurs produits, l'expérience et le sens, entre la subjectivité individuelle et la collectivité, et redéfinit le rôle des acteurs sociaux en étudiant les modes spécifiques de production du sens dans un art comme la danse.

St-Pierre, Julie. Le Conte en contexte : ethnographie de la pratique du conte en famille dans le Québec contemporain. Thèse (Ph. D.), Université du Québec à Montréal, 2011, 1-317 p. [Direction : Rachel Bouvet].

L'omniprésence du conte dans toutes les cultures en a fait un objet universel transportant des significations propres à l'imaginaire commun des sociétés. Les nombreuses manifestations contemporaines de la persistance du conte dans les différentes sphères de la réalité nous obligent en effet à nous demander comment celui-ci réussit encore à s'immiscer aussi profondément dans nos vies et à y créer du sens. L'étude que nous proposons, en s'appuyant sur les bases théoriques déjà établies, cherche à réintégrer la nécessité pragmatique du conte au cœur de sa signification. À travers la démarche du terrain ethnographique réalisé auprès de vingt et une familles québécoises, l'étude révèle cette forme narrative comme une pratique du quotidien qui rythme la vie familiale. L'exploration des dimensions sensible, rituelle, récréative et sociale de la pratique permet de comprendre comment celles-ci opèrent avec le récit pour lui donner une signification particulière. Aussi, le livre comme objet rituel matérialisant le passage de la réalité à l'univers de la fiction revêt une importance capitale sur la pratique familiale dans le Québec contemporain. Ce dernier s'avère même, dans certaines familles, indispensable à son exercice. Par l'animation du livre de conte, le parent construit un récit sémiotiquement métissé qui veut transmettre le goût de la lecture à l'enfant. En conclusion, la perspective de recherche originale prônée ici parvient à la démonstration que le conte s'inscrit effectivement aux fondements anthropologiques du récit, non seulement parce qu'il possède une structure ou un symbolisme universels, mais surtout parce qu'il suppose un contexte d'interaction au cœur de la relation humaine. 